\title{
Interval-valued Analytic Network Process and its Application in Risk Assessment of Dynamic Alliance
}

\author{
Xiao-Guang Zhou*, Yan-Hui Zhou and Xiao-Xiao Cui \\ Dongling School of Economics and Management, University of Science and \\ Technology Beijing, Beijing 100083, China \\ *Corresponding author E-mail: xiaoguang@ustb.edu.cn
}

\begin{abstract}
Analytic Network Process (ANP) has been widely used in solving many complicated decision making problems. An approach to acquire the comprehensive weights of interval-valued Analytic Network Process (IV-ANP) is proposed. Firstly, the independent local interval-valued weights are obtained by solving the goal programming for intervalvalued pairwise comparison matrices. Based on the interaction and feedback relationships among criteria and/or indices, an unweighted interval-valued supermatrix is formed. To avoid loss of information, an extreme value method is developed for acquiring the global weights of IV-ANP. Secondly, the alternatives are ranked on the basis of interval-valued preference order. Finally, with the interaction and feedback relationships between criteria and/or indices being considered, an index system for assessing the risk of dynamic alliance is presented. With the uncertain and inaccurate information during the evaluation process being considered, the proposed IV-ANP method is applied for risk assessment of dynamic alliance, and sensitivity analyses for some key risk factors are performed to show their impacts on the risk levels of alliance enterprises.
\end{abstract}

Keywords: Analytic Network Process (ANP), interval-valued Analytic Network Process (IV-ANP), dynamic alliance, risk assessment, goal programming

\section{Introduction}

Due to its adaptation to environmental change and market competition, the organizational form of dynamic alliance has been developing rapidly in recent years. However, many fail, while an even greater proportion perform poorly. Recent estimates put the failure rate of alliances between $60 \%$ and $70 \%$, indicating companies that pursue alliances are likely to fail [1]. Although such failures may be for many reasons - poor partner selection and poor alliance management are two general causes [2]. Many problems on dynamic alliance, such as partner selection, operation management, information transmission and exchange, and so on, have been investigated. Unfortunately, the risk management of dynamic alliance has not received deserved attention [3]. The risk analysis of dynamic alliance will help enterprises to make the risk management plan and to keep the stability of the alliance. This paper focuses on risk assessment, which is one of the most important topics of risk management for dynamic alliance.

To reduce the risk and achieve successful alliance, (1) some scholars analyzed the factors which impact the success of dynamic alliance. For example, Venkatesh et al. investigated two questions: under what market-driven characteristics should either brand manufacturer forge or sustain the alliance; what product market characteristics should the alliance promoter seek or alter to increase its payoffs from the alliance [4]. Chen discussed the effects of environment and partner characteristics on the choice of alliance forms [5]. Rothaermel and Deeds developed a model that links differential demands of alliance type and the benefits of alliance experience to an observable outcome from a firm's alliance management capability [6]. They found that alliance type and alliance 
experience moderate the relationship between a high-technology venture's $R \& D$ alliances and its new product development. Delerue and Simon pointed out that cross-cultural interactions are becoming important in risk perceptions [7]. Mukherjee et al. identified that environmental uncertainty and knowledge intensity impede firms' $R \& D$ alliance formation; the focal firm's overall trust in partners enhances alliance formation in small to medium-sized enterprises [8], etc., (2) Some scholars discussed how to manage the risk of dynamic alliance, such as Das and Teng introduced a model of dynamic alliance with managerial risk perception as its core [9]. Rosenkranz and Schmitz discussed the dynamic evolution of property rights regimes in $\mathrm{R} \& \mathrm{D}$ alliances by applying the incomplete contract approach [10]. Ip, et al., presented a risk-based partner selection method to select partner, and proposed a rule-based genetic algorithm to ensure the due date of a project in dynamic alliance [11]. Huang, et al., developed a two-level distributed decision making model for the risk management of dynamic alliance, which describe the decision processes of the owner and the partners of the dynamic alliance, respectively [12]. Lee, et al., illustrated the locus of dynamic knowledge articulation and capabilities development by exploring drivers of dynamic learning in service alliance enterprises [13], etc., (3) A few scholars conducted a risk assessment for dynamic alliance. For instance, Huang, et $a l$., developed a fuzzy synthetic evaluation embedded nonlinear integer programming model of risk programming for dynamic alliance [14]. Li and Liao proposed an evaluation approach based on the framework of the evidential reasoning [3], etc.

In the existing research literatures of risk assessment for dynamic alliance, however, the interaction and feedback relationships between criteria and/or indices are not completely considered. Saaty introduced ANP in 1996 [15], which is the extension of AHP (Analytic Hierarchy Process). As its conformation and adaptation, AHP/ANP has been widely used as a multi-criteria decision analysis tool in many fields [16], such as project selection [17], risk evaluation [18-21], etc. The hierarchical decision structure is introduced to incorporate interdependence and feedback effect in the prioritization of the selected criteria and/or indices.

During the risk evaluation process, there is lots of imprecise and uncertainty information. The crisp pairwise comparison seems to be insufficient to describe the judgments of experts or decision-makers. Therefore, the AHP/ANP-based decision model seems to be ineffective in dealing with the inherent fuzziness or uncertainty for judgment during the pairwise comparison process [22]. Although the use of the discrete scale of 1-9 to represent the verbal judgment in pairwise comparisons has the advantage of simplicity, it does not take into account the uncertainty associated with the mapping of one's perception or judgment to a number. In common practice, the experts or decision makers might be uncertain about their own level of preference. Due to incomplete information or knowledge, uncertainty and complexity within the decision making environment, experts or decision-makers also tend to designate their preferences in the form of natural language expressions which are most often vague and uncertain. Such situations will take place when assessing the risk of dynamic alliance.

Consequently, it's more appropriate to make risk evaluation and management scheme under interval-valued or fuzzy condition. Zhou and Lu proposed a fuzzy ANP-based method to make up for the deficiency in the conventional risk assessment process for dynamic alliance [22]. To provide a new evaluation method for decision makers or stakeholders, the objective of this paper is to present an IV-ANP method for risk evaluation of dynamic alliance. 


\section{Preliminary Knowledge}

\subsection{ANP}

The decision making problem is structured hierarchically at different levels in the AHP, composed of finite number of elements (criteria or alternatives). The relative importance of the decision elements (weights of the criteria and scores of the alternatives) is assessed indirectly from pairwise comparison judgments. The empirical effectiveness and theoretical validity of the AHP and its flaws have been discussed by many authors $[23,24]$. Consequently, ANP is presented by Saaty. ANP is the first mathematical theory that makes it possible to deal with all kinds of dependences and feedbacks by replacing hierarchies with networks. The local priorities in ANP are established in the same manner as they are in AHP using pairwise comparisons and judgments. However, the supermatrix approach which became popularly known as the ANP approach is becoming an attractive tool to understand more of the complex decision problem as it overcomes the limitation of the AHP's linear hierarchy structure [25].

In method ANP, it can be divided into three levels: control layer (includes target and decision-making rules); network layer and alternative layer which composed by decisionmaking object (scheme, enterprise etc.). In control level, all decision-making rules are thought to be independent with each other, and they are just influenced by target element; there could be no decision-making rules in control level, but one target at least, as shown in Figure 1. Generally, if criteria set $A$ has an effect on set $B$ then a line with arrow from $A$ to $B$ is added. If the criteria set $A$ have interaction itself then $A$ is inner dependence, and an arc with arrow is added to set $A$.

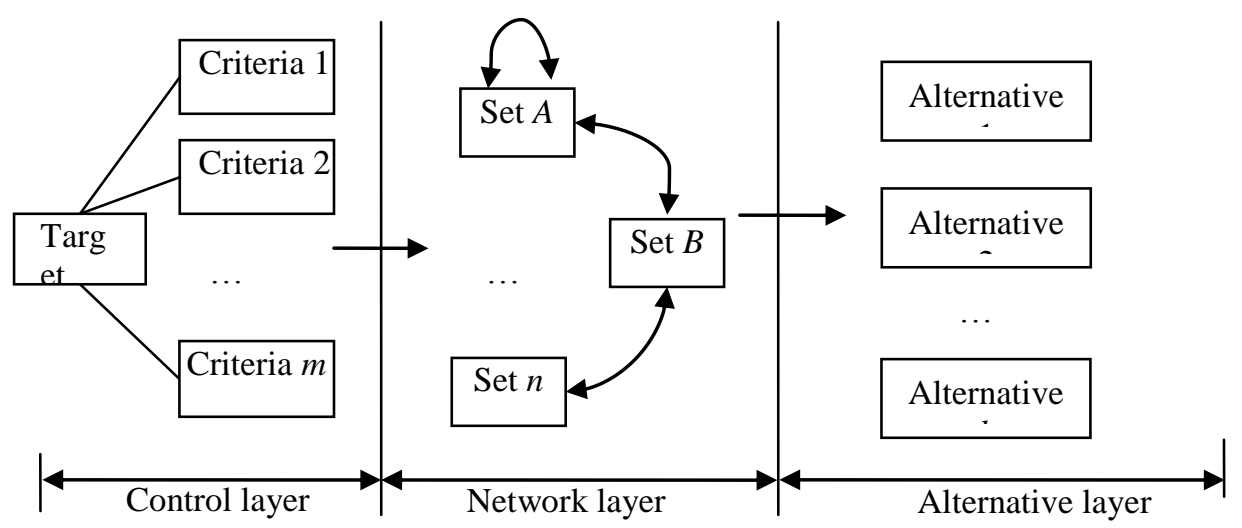

Figure 1. ANP Model

The supermatrix was introduced to serve as a unifying framework for the study of priorities in hierarchy and in systems with feedback. Consider a system that can be decomposed into m clusters $C_{1}, C_{2}, \ldots, C_{m}$, and let the elements in $C_{k}$ having $n_{k}$ elements be denoted as $e_{k_{1}}, e_{k_{2}}, \ldots, e_{k_{n k}}$. Then, a supermatrix representation of this system can be viewed as a partitioned matrix that describes the interaction between the elements and clusters of a system, as shown in Figure 2. In this supermatrix, the block matrix contains a column of priority weights which represent the impact of all elements in the ith cluster on each of the elements in the $j$ th cluster. 


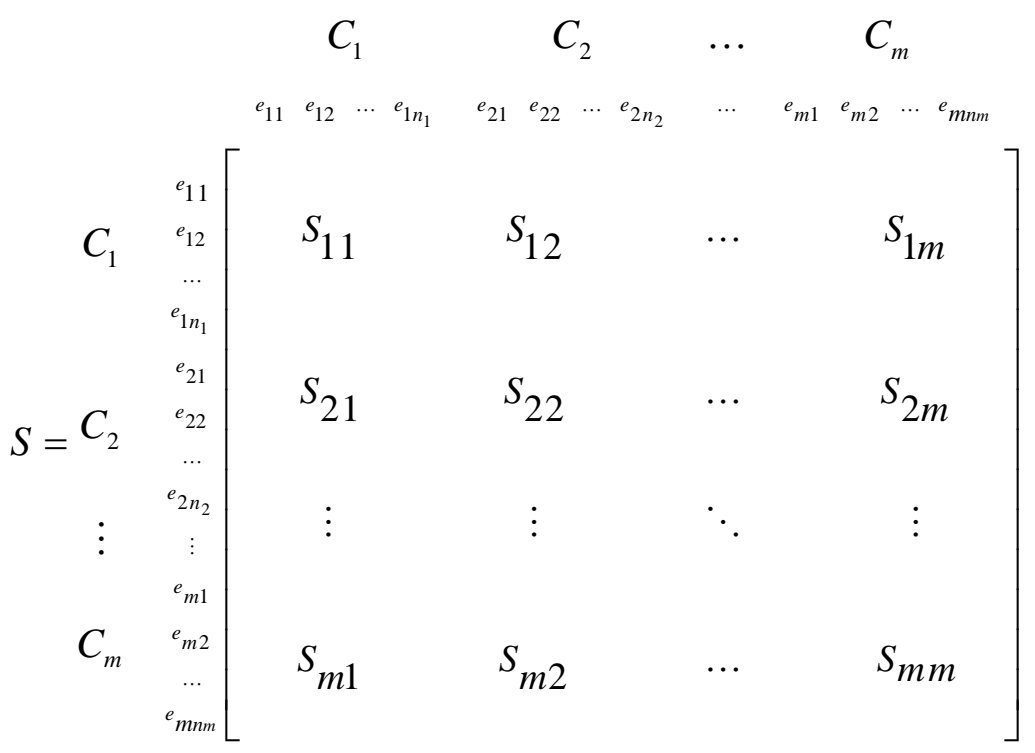

Figure 2. Supermatrix of ANP System

Formula (1) is an example of block matrix of the supermatrix. Some of its entries may be zero corresponding to those elements which have no path of direct interaction (or influence) to other elements. Raising a matrix to exponential powers gives the long term relative influences of the elements on each other [26].

$$
S_{i j}=\left[\begin{array}{cccc}
W_{i_{1}\left(j_{1}\right)} & W_{i_{1}\left(j_{2}\right)} & \ldots & W_{i_{1}\left(j_{n_{j}}\right)} \\
W_{i_{2}\left(j_{1}\right)} & W_{i_{2}\left(j_{2}\right)} & \ldots & W_{i_{2}\left(j_{n_{j}}\right)} \\
& & \ddots & \\
W_{i_{n_{j}}\left(j_{1}\right)} & W_{i_{n_{j}}\left(j_{2}\right)} & \ldots & W_{i_{n_{j}}\left(j_{n_{j}}\right)}
\end{array}\right]
$$

\subsection{Goal Programming Method for Solving an Interval-valued Judgment Matrix}

A number of methods have been developed to handle the local weights of intervalvalued comparison matrices, such as fuzzy programming method [27], eigenvector method [28], upper approximation [29], interval regression analysis [30] and goal programming method [31]. Goal programming method, as a reasonable and effective means, is adopted in this study. The basic theory of the method is as follows [31]

Assume that decision makers can provide the index $i$ is $l_{i j}$ to $u_{i j}$ times as important as the index $j$ during the process of pairwise comparison. The parameters $l_{i j}$ and $u_{i j}$ are nonnegative real numbers, and $l_{i j} \leq u_{i j}$. For the interval-valued comparison matrix $A$, there should exist a normalized interval weight vector $\left.W=\left(\left[w_{1}{ }^{L}, w_{1}{ }^{U}\right]\right),\left[w_{2}{ }^{L}, w_{2}{ }^{U}\right]\right), \ldots,\left[w_{n}{ }^{L}\right.$, $\left.\left.w_{n}{ }^{U}\right]\right)^{T}$. For all $i, j=1,2, \ldots, n ; i \neq j, a_{i j}=\left[l_{i j}, u_{i j}\right] \approx\left[w_{i}^{L}, w_{i}^{U}\right] /\left[w_{j}^{L}, w_{j}^{U}\right]$. The normalized interval-valued weight vector $W$ satisfies:

$$
\begin{gathered}
w_{i}^{L}+\sum_{j=1, j \neq i}^{n} w_{j}^{U} \geq 1, \quad i=1,2, \ldots, n, \\
w_{i}^{U}+\sum_{j=1, j \neq i}^{n} w_{j}^{L} \leq 1, \quad i=1,2, \ldots, n .
\end{gathered}
$$

If the interval-valued judgment matrix $A$ is the precise comparison about the interval weight vector $W$, this expression $a_{i j}=\left[l_{i j}, u_{i j}\right]=\left[w_{i}^{L}, w_{i}^{U}\right] /\left[w_{j}^{L}, w_{j}^{U}\right]$ stands. According to the division operation rule on interval numbers, i. e. $\left[b_{L}, b_{U}\right] /\left[d_{L}, d_{U}\right]=\left[b_{L} / d_{U}, b_{U} / d_{L}\right]$, where $\left[b_{L}, b_{U}\right]$ and $\left[d_{L}, d_{U}\right]$ are two positive interval numbers, the upper and lower bounds of interval-value judgment matrix have: 
where $W_{L}=\left(w_{1}{ }^{L}, \ldots, w_{n}{ }^{L}\right)$ and $W_{U}=\left(w_{1}{ }^{U}, \ldots, w_{n}{ }^{U}\right)^{T}$.

$$
A_{L} W_{U}=W_{U}+(n-1) W_{L},
$$

Due to the presence of subjectivity and uncertainty, the DM's judgments are not always accurate. Therefore, Equations. (4) and (5) may not hold precisely. Based on such an analysis, the following deviation vectors are introduced:

$$
\begin{aligned}
& E=\left(A_{L}-I\right) W_{U}-(n-1) W_{L}, \\
& \Gamma=\left(A_{U}-I\right) W_{L}-(n-1) W_{U},
\end{aligned}
$$

where $E=\left(\varepsilon_{1}, \varepsilon_{2}, \ldots, \varepsilon_{n}\right)^{T}, \Gamma=\left(\gamma_{1}, \gamma_{2}, \ldots, \gamma_{n}\right)^{T}$, and $I$ is a $n$ by $n$ unit matrix whose elements on the leading diagonal are 1 , and all the other elements are 0 . Let

$$
\begin{aligned}
& \varepsilon_{i}^{+}=\frac{\varepsilon_{i}+\left|\varepsilon_{i}\right|}{2}, \quad \varepsilon_{i}^{-}=\frac{-\varepsilon_{i}+\left|\varepsilon_{i}\right|}{2}, i=1,2, \ldots, n ; \\
& \gamma_{i}^{+}=\frac{\gamma_{i}+\left|\gamma_{i}\right|}{2}, \gamma_{i}^{-}=\frac{-\gamma_{i}+\left|\gamma_{i}\right|}{2}, i=1,2, \ldots, n .
\end{aligned}
$$

Accordingly, the optimization model for solving the local interval-valued weights is:

$$
\begin{aligned}
& \min J=\sum_{i=1}^{n}\left(\varepsilon_{i}^{+}+\varepsilon_{i}^{-}+\gamma_{i}^{+}+\gamma_{i}^{-}\right)=e^{T}\left(E^{+}+E^{-}+\Gamma^{+}+\Gamma^{-}\right) \\
& \text {s.t. }\left\{\begin{array}{l}
\left(A_{L}-I\right) W_{U}-(n-1) W_{L}-E^{+}+E^{-}=0, \\
\left(A_{U}-I\right) W_{L}-(n-1) W_{U}-\Gamma^{+}+\Gamma^{-}=0, \\
w_{i}^{L}+\sum_{j=1, j \neq i}^{n} w_{j}^{U} \geq 1, \quad i=1,2, \ldots, n, \\
w_{i}^{U}+\sum_{j=1, j \neq i}^{n} w_{j}^{L} \leq 1, \quad i=1,2, \ldots, n, \\
W_{U}-W_{L} \geq 0, \\
W_{L}, W_{U}, E^{+}, E^{-}, \Gamma^{+}, \Gamma^{-} \geq 0 .
\end{array}\right.
\end{aligned}
$$

\subsection{The Preference Relation of Interval Numbers}

Let $a=\left[a_{1}, a_{2}\right]$ and $b=\left[b_{1}, b_{2}\right]$ be two interval weights, we refer to the degree of one interval weight being greater than another one as the degree of preference. Accordingly, we have the following definitions and properties.

Definition 1 [32] The degree of preference of interval-valued $a$ over $b$ (or $a>b$ ) is defined as

$$
P(a>b)=\frac{\max \left(0, a_{2}-b_{1}\right)-\max \left(0, a_{1}-b_{2}\right)}{\left(a_{2}-a_{1}\right)+\left(b_{2}-b_{1}\right)}
$$

The degree of preference of interval-valued $b$ over $a$ (or $b>a$ ) can be defined in the same way. That is,

$$
P(b>a)=\frac{\max \left(0, b_{2}-a_{1}\right)-\max \left(0, b_{1}-a_{2}\right)}{\left(a_{2}-a_{1}\right)+\left(b_{2}-b_{1}\right)}
$$

Obviously, $P(a>b)+P(b>a)=1$, and $P(a>b)=P(b>a)=0.5$, when $a=b$ (that is $a_{1}=$ $\left.b_{1}, a_{2}=b_{2}\right)$.

Definition 2 [32] If $P(a>b)>P(b>a)$, then $a$ is said to be superior to $b$ at the degree of $P(a>b)$

$P(a>b)$, denoted by $a \stackrel{P(a>b)}{\succ} b$; If $P(a>b)=P(b>a)=0.5$, then $a$ is said to be equal to $b$, denoted by $a \square b$; If $P(b>a)>P(a>b)$, then $a$ is said to be inferior to $b$ at the degree of 
$P(a>b)$, denoted by $a \stackrel{P(b>a)}{\prec} b$.

It is easy to prove the following properties.

Property 1. If and only if $a_{1} \geq b_{2}$, then $P(a>b)=1$.

Property 2. If $a_{1} \geq b_{1}$ and $a_{2} \geq b_{2}$, then $P(a>b) \geq 0.5$ and $P(b>a) \leq 0.5$.

Property 3. If $P(a>b) \geq 0.5$ and $P(b>c) \geq 0.5$, then $P(a>c) \geq 0.5$.

\section{IV-ANP Method}

To acquire interval-valued weights, an IV-ANP framework is proposed as follows.

\subsection{Calculate the Local Interval-valued Weights}

First of all, according to the interaction and feedback relationships among criteria and/or indices, the elements of control layer and network layer are determined, and the ANP network structure is established. Based on the opinions of experts or decision makers, the interval-valued pairwise comparison matrices between the criteria and/or indices are formed. The preferences of experts or decision makers can be expressed by interval numbers. The local weights of the interval-valued judgment matrices can be achieved by Eq. (10).

\subsection{Calculate the Comprehensive Interval-valued Weights}

The most existing researches for solving the comprehensive interval-valued weights are to simplify the interval value to a single value. Such method has the advantage of simple, but to some extent the loss of information will offset the advantage [31]. Although some scholars have studied the interval-valued AHP method [29-35], few scholars research the interval-valued ANP method so far. Vahdani, et al., developed an intervalvalued fuzzy ANP to solve multi-criteria decision making problems [36]. In their proposed methodology, the triangular interval-valued fuzzy weights are achieved by logarithmic least squares method. Only the triangular interval-valued fuzzy weights for the relative importance and the interdependence priorities of the criteria are used. That is, they did not form the unweighted and weighted supermatrix, and they did not acquire the global weights through interval-valued ANP. Therefore, in this paper, an extreme value method for solving the comprehensive weights of interval-valued ANP is proposed based on the interaction and feedback relationships among criteria and/or indices.

The main idea of the extreme value method is as follows. The element $q_{i j}$ in the unweighted supermatrix indicates a local weight for factor $C_{i}$ to $C_{j}$, and the corresponding element $q_{i j}$ in the convergent supermatrix indicates a global weight for the factor in the whole system. If an unweighted supermatrix is composed of the upper bound of element $q_{i j}$ and lower bounds of other elements, then the global weight of the element $q_{i j}{ }^{\prime}$ indicates great importance of the element but less importance of other elements. The global weights can be acquired by normalizing the unweighted supermatrix and solving the convergence of the weighted supermatrix. Therefore, the global weight of the element $q_{i j}{ }^{\prime}$ can be used as the upper bound of the final interval-valued weight. In the same way, the lower bound of the final interval-valued weight of the element $q_{i j}{ }^{\prime}$ can be achieved.

The implementation steps are as follows:

(1) Adding the local interval-valued weights into the unweighted supermatix $\left(Q_{i j}\right)_{n \times n}$. The column vectors of $Q_{i j}$ indicate the influence degree of the element $C_{i}$ to $C_{j}$. If the element $C_{i}$ have no impact on $C_{j}$, then $Q_{i j}=0$.

(2) The unweighted supermatrix $\left(Q_{i j}\right)_{n \times n}$ can be divided into two supermatrices: one is the lower bound value of the interval-valued weight, named as $Q_{L}$; the other is the upper bound value of the interval-valued weight, named as $Q_{U}$.

(3) Exchange the $i$ th line of $Q_{L}$ with the $i$ th line of $Q_{U}(i=1,2, \ldots, n$.), then two new 
matrices, named $Q_{L i}$ and $Q_{U i}$ can be gained.

(4) The weighted supermatrix $Q_{L i}{ }^{\prime}$ and $Q_{U i}{ }^{\prime}$ are achieved by normalizing the $Q_{L i}$ and $Q_{U i}$ individually.

(5) The lower limit supermatrix $Q_{L i}{ }^{\prime \prime}$ and the upper limit supermatrix $Q_{U i}{ }^{\prime \prime}$ are calculated by

$$
Q_{L i}^{\prime \prime}=Q_{L i}^{\prime \infty}=\lim _{t \rightarrow \infty} Q_{L i}^{\prime t}, Q_{U i}^{\prime \prime}=Q_{U i}^{\prime \infty}=\lim _{t \rightarrow \infty} Q_{U i}^{\prime t} .
$$

A column randomly from $Q_{L i}{ }^{\prime \prime}$ is selected as the lower of final comprehensive weights, and a column from $Q_{U i}{ }^{\prime \prime}$ is selected as the upper of the final comprehensive weights.

The advantages of this method are as follows.

(1) It achieves interval-valued weights and avoids the loss of information from interval-valued weights to single values.

(2) The final interval-valued weights cover all the extreme conditions so that it has strong comprehensiveness.

(3) The calculation is simple as well. Compared with the method simplified to a single value for $\mathrm{n}$ risk factors, the calculation only increases $2 n$ times. It doesn't improve the complexity and operation time too much.

\subsection{Select the optimal alternative}

To rank the alternatives, the scores of alternatives need to be calculated. Assume $R_{i}$ is the score of $i$ th alternative, then $R_{i}$ can be achieved by

$$
R_{i}=\sum_{i=1}^{J} \sum_{k=1}^{K_{j}} P_{j} A_{k j}^{D} A_{k j}^{I} S_{i k j}
$$

where $P_{j}$ is relative importance weight of dimension $j$ on final goal; $A_{k j}^{D}$, relative importance weight for attribute-enabler $k$ of dimension $j$, and for the dependency $(D)$ relationships within attribute-enabler's component level; $A_{k j}^{I}$, stabilized relative importance weight for attribute -enabler $k$ of dimension $j$, and for the independency $(I)$ relationships within attribute-enabler's component level; $S_{i k j}$, relative impact of alternative $i$ on attribute-enabler $k$ of dimension $j$ of selection network; $K_{j}$, index set of attributeenablers for dimension $j$; and $J$ is index set for attribute $j$.

Then the alternatives can be ranked by preference relation of interval-value order. The preference degree $P_{i j}$ can be acquired by

$$
P_{i j}=P\left(w_{i}>w_{j}\right)=\frac{\max \left(0, w_{i}^{U}-w_{j}^{L}\right)-\max \left(0, w_{i}^{L}-w_{j}^{U}\right)}{\left(w_{i}^{U}-w_{i}^{L}\right)+\left(w_{j}^{U}-w_{j}^{L}\right)},
$$

where $i, j=1,2, \ldots, n ; i \neq j$.

\section{Case Study}

Suppose there are four large-scale manufacturing enterprises, named as $E_{1}, E_{2}, E_{3}$ and $E_{4}$. To catch the market opportunity and win the market competition, they have being formed a dynamic alliance. To make risk management plan and keep the alliance stability, and to make more reasonable income distribution scheme as well, an expert committee is formed to evaluate the risk of the dynamic alliance. The proposed IV-ANP method for evaluating the risk level comprises the following process.

\subsection{The Index System of Risk Evaluation for Dynamic Alliance}

According to the source and properties of this dynamic alliance, the risk of dynamic alliance can be divided into five parts: identification risk, technology risk, cooperation risk, operation risk and environment risk. It includes 16 sub-criteria, and the dependence and interaction relations of indices are as shown in Figure 3. 


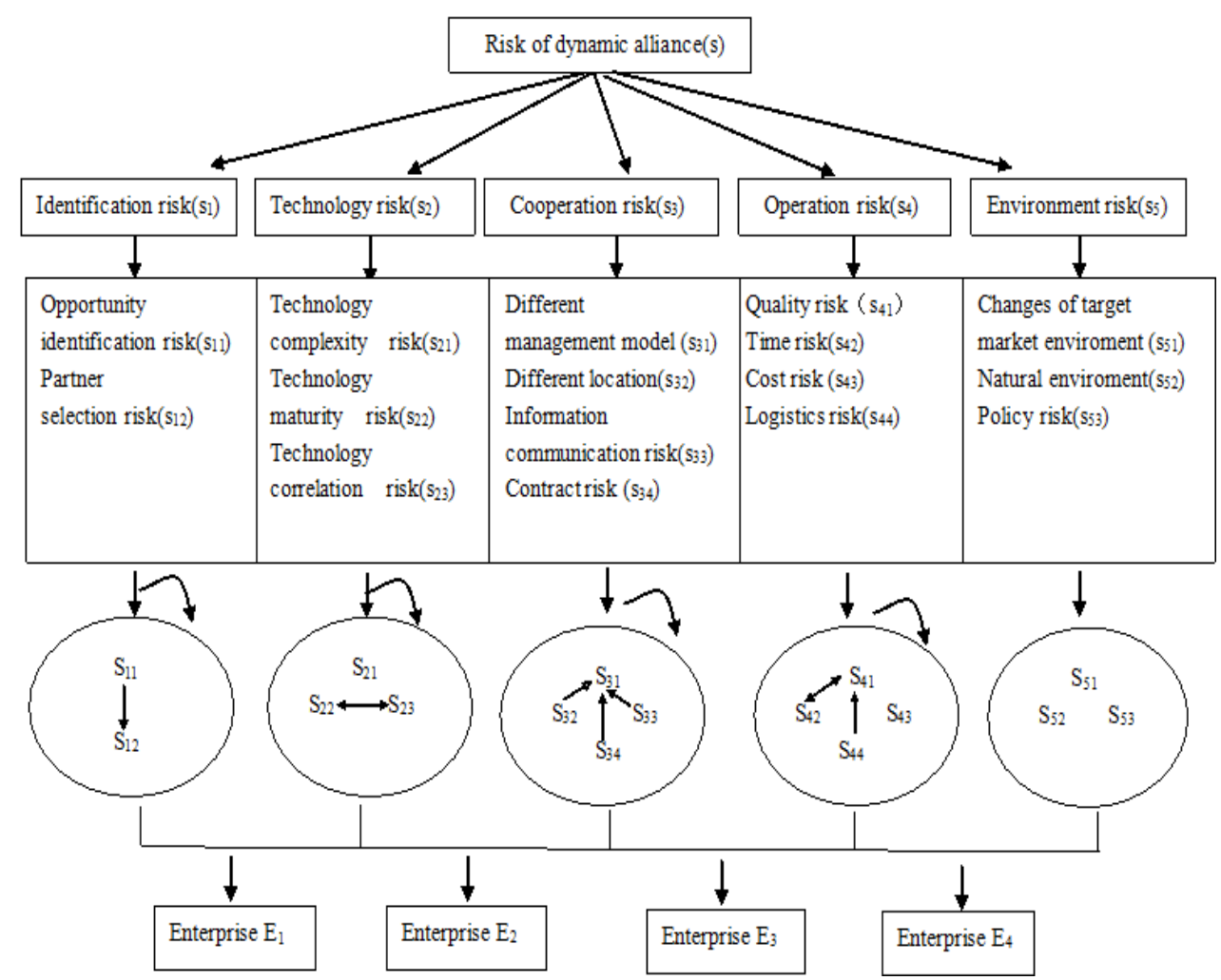

Figure 3. The Index System of Risk Evaluation for Dynamic Alliance

\subsection{Risk Assessment of Dynamic Alliance based on IV-ANP}

The risk evaluation process of dynamic alliance based on IV-ANP includes the following steps:

Step 1: With the interaction and feedback relationships among criteria and/or subcriteria being considered, a four-level index system is proposed, as shown in Figure 3. Pairwise comparison matrices among dimensions and/or attributes and the scores of alternatives are determined by the decision committee.

Step 2: According to Eq. (10), the local interval-valued weights are obtained. For example, Table 1 shows the pairwise comparison matrix for the quality risk $\left(S_{41}\right)$, time risk $\left(S_{42}\right)$, cost risk $\left(S_{43}\right)$ and logistics risk $\left(S_{44}\right)$ at the dimension of environment $\operatorname{risk}\left(S_{5}\right)$ in the control layer and the sub-dimension of target market environment change $\operatorname{risk}\left(S_{51}\right)$. On the basis of goal programming, with the help of Matlab, the local weights are $(0.0592$, $0.0628,[0.5947,0.6263],[0.2517,0.2833])$ respectively. Here single value means that the upper bound and the lower bound of the interval value are equal. The local interval-valued weights of other judgment matrices are achieved in the same manner.

Table 1. Comparison Matrix and Local Weights for Operation Risk at the Dimension of $S_{5}$ and Sub-dimension of $S_{51}$

\begin{tabular}{cccccc}
\hline$S_{51}$ & $S_{41}$ & $S_{42}$ & $S_{43}$ & $S_{44}$ & $w$ \\
\hline$S_{41}$ & 1 & 1 & {$[1 / 4,1 / 3]$} & {$[1 / 8,1 / 7]$} & 0.0592 \\
$S_{42}$ & 1 & 1 & {$[1 / 4,1 / 3]$} & {$[1 / 6,1 / 5]$} & 0.0628 \\
$S_{43}$ & {$[3,4]$} & {$[3,4]$} & 1 & {$[5,6]$} & {$[0.5947,0.6263]$} \\
$S_{44}$ & {$[7,8]$} & {$[5,6]$} & {$[1 / 6,1 / 5]$} & 1 & {$[0.2517,0.2833]$} \\
\hline
\end{tabular}

Step 3: The unweighted supermatrix is formed by adding all the local interval-valued weights. 
Step 4: The weighted supermatrix and limit supermatrix can be calculated by the proposed extreme method.

Table 2. The Lower Bound of the Comprehensive Weights

\begin{tabular}{|c|c|c|c|c|c|c|c|c|c|c|c|c|c|}
\hline Index & $P_{j}$ & $A_{k j}^{D}$ & $A_{k j}^{I}$ & $w$ & $w^{\prime}$ & $S_{1 k j}$ & $S_{2 k j}$ & $S_{3 k j}$ & $S_{4 k j}$ & $D_{1}$ & $D_{2}$ & $D_{3}$ & $D_{4}$ \\
\hline$S_{11}$ & 0.5453 & 0.8571 & 0.0799 & 0.0373 & 0.5814 & 0.0605 & 0.3851 & 0.2245 & 0.1820 & 0.0352 & 0.2239 & 0.1305 & 0.1058 \\
\hline$S_{12}$ & 0.5453 & 0.1250 & 0.0487 & 0.0033 & 0.0517 & 0.1058 & 0.0990 & 0.3269 & 0.3023 & 0.0055 & 0.0051 & 0.0169 & 0.0156 \\
\hline$S_{21}$ & 0.1742 & 0.3747 & 0.1137 & 0.0074 & 0.1155 & 0.1126 & 0.1257 & 0.3401 & 0.2805 & 0.0130 & 0.0145 & 0.0393 & 0.0324 \\
\hline$S_{22}$ & 0.1742 & 0.2058 & 0.0720 & 0.0026 & 0.0402 & 0.1312 & 0.1146 & 0.2611 & 0.3178 & 0.0053 & 0.0046 & 0.0105 & 0.0128 \\
\hline$S_{23}$ & 0.1742 & 0.2084 & 0.0532 & 0.0019 & 0.0301 & 0.0232 & 0.2457 & 0.4608 & 0.1655 & 0.0007 & 0.0074 & 0.0139 & 0.0050 \\
\hline$S_{31}$ & 0.1170 & 0.0605 & 0.0099 & 0.0001 & 0.0011 & 0.0288 & 0.2483 & 0.4608 & 0.1659 & 0.0000 & 0.0003 & 0.0005 & 0.0002 \\
\hline$S_{32}$ & 0.1170 & 0.3851 & 0.0697 & 0.00 & 0.048 & 0.44 & 0.19 & 0.2029 & 0.09 & 0.0219 & 0.0097 & 0.00 & 0.0046 \\
\hline$S_{33}$ & 0.1170 & 0.2245 & 0.0560 & 0.00 & 0.0229 & 0.4 & 0.29 & 0.1 & 0.03 & & 0.0067 & 0.00 & 0.0008 \\
\hline$S_{34}$ & 0.1170 & 0.1820 & 0.0360 & 0.000 & 0.0119 & 0.668 & 0.17 & 0.10 & 0.02 & 0.0080 & 0.0021 & 0.0012 & 0.0003 \\
\hline$S_{41}$ & 0.0166 & 0.1058 & 0.0162 & 0.0000 & 0.0004 & 0.019 & 0.1727 & 0.5827 & 0.1647 & 0.0000 & 0.0001 & 0.0003 & 0.0001 \\
\hline$S_{42}$ & 0.0166 & 0.0990 & 0.0342 & 0.0001 & 0.0009 & 0.4857 & 0.2312 & 0.0975 & 0.0535 & 0.0004 & 0.0002 & 0.0001 & 0.0000 \\
\hline$S_{43}$ & 0.016 & 0.3269 & 0.0658 & 0.000 & 0.0056 & 0.6404 & 0.1972 & 0.1010 & 0.0229 & 0.0036 & 0.0011 & 0.0006 & 0.0001 \\
\hline$S_{44}$ & 0.01 & 0.302 & & م & 0.0037 & & & 0.14 & 0.03 & 0.00 & 0.00 & 0.00 & 0.0001 \\
\hline$S_{51}$ & 0.11 & & & & & & & & & & & & 0.0074 \\
\hline$S_{52}$ & 0.110 & 102 & 0.0611 & & & & & & 0.0 & & & & 0.0012 \\
\hline \multirow[t]{2}{*}{$S_{53}$} & 0.1100 & .2040 & 0.0164 & 0.0004 & 0.0057 & 0.4554 & 0.2578 & 0.1195 & 0.0762 & 0.0026 & 0.0015 & 0.0007 & 0.0004 \\
\hline & & & fin & l score & & & & & & 0.1419 & 0.2902 & 0.2506 & 0.1868 \\
\hline
\end{tabular}

Step 5: According to the Eq. (14), the risk ratings of the candidates with respect to indices can be calculated, as shown in Tables 2 and 3. Where $w^{\prime}$ is the normalization of $w$; and $w$ is the global weights of indices; $D$ is the scores of alternatives with regard to indices.

Table 3. The Upper Bound of the Comprehensive Weights

\begin{tabular}{|c|c|c|c|c|c|c|c|c|c|c|c|c|c|}
\hline Index & $P_{j}$ & $A_{k j}^{D}$ & $A_{k j}^{I}$ & $w$ & $w^{\prime}$ & $S_{1 k j}$ & $S_{2 k j}$ & $S_{3 k j}$ & $S_{4 k j}$ & $D_{1}$ & $D_{2}$ & $D_{3}$ & $D_{4}$ \\
\hline$S_{11}$ & & 875 & 1053 & 0502 & 4366 & 0.0646 & 0.4669 & 0.2864 & 0.2839 & 0.0282 & 0.2038 & 0.1250 & 0.1240 \\
\hline$S_{12}$ & & & & & & & & & & & & & \\
\hline$S_{21}$ & & & & & & & & & & & & & 0.0559 \\
\hline$S_{22}$ & & & & & & & & & & & & & 0.0179 \\
\hline$S_{23}$ & & & & & & & & & & & & & 0.0162 \\
\hline$S_{31}$ & 1 & 0.06 & & & & & & & & & & & 0.0002 \\
\hline$S_{32}$ & 011 & 0.466 & & & & & & & & & & & 0.0039 \\
\hline$S_{33}$ & 1 & 0.28 & & & & & & & & & & & 0.0020 \\
\hline$S_{34}$ & & & & & & & & & & & & & 0.0004 \\
\hline$S_{41}$ & & & & & & & & & & & & & 0.0002 \\
\hline$S_{42}$ & 0.0281 & 0.16 & 0.0503 & & & & & & 0.09 & & & & 0.0002 \\
\hline$S_{43}$ & 0.0281 & 0.492 & 0.1005 & & & 0.6668 & & & 0.03 & 0.0081 & & & 0.0004 \\
\hline$S_{44}$ & 0.028 & 021 & 0.069 & & & & & & & 0.0 & & & 0.0005 \\
\hline$S_{51}$ & 0.18 & & & & & & & & & & & & 0.0136 \\
\hline$S_{52}$ & & & & & & & & & & & & & 0.0025 \\
\hline \multirow[t]{2}{*}{$S_{53}$} & 101 & 0.16 & 0.0230 & 0.000 & 0. & 0401 & 0.337 & 0.145 & 0.10 & 0.0030 & & 0.0009 & 0.0006 \\
\hline & & & & & & & & & & 0.1862 & 0.3172 & 0.3423 & 0.2534 \\
\hline
\end{tabular}

According to Tables 2 and 3, the risk scores $W_{i}(i=1,2,3,4)$ of the four enterprises can be obtained, as shown in Table 4.

Table 4. The Risk Scores of the Four Enterprises

\begin{tabular}{ccccc}
\hline$W$ & $W_{1}$ & $W_{2}$ & $W_{3}$ & $W_{4}$ \\
\hline$w_{i}{ }^{L}$ & 0.1419 & 0.2902 & 0.2506 & 0.1868 \\
$w_{i}{ }^{U}$ & 0.1862 & 0.3172 & 0.3423 & 0.2534 \\
\hline
\end{tabular}


Step 6: Rank the risk level of the four enterprises using the method of interval-valued preference order relation. The preference degree of the interval-valued weights can be calculated by Eq. (15). Their preference order relations are described in Figure 4.

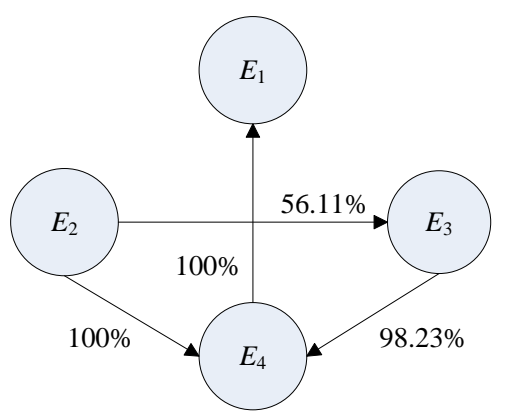

Figure 4. The Preference Order Relations

According to Figure 4, the risk preference relations of the four enterprises are:

$$
W_{2} \stackrel{56.11 \%}{\succ} W_{3} \stackrel{98.23 \%}{\succ} W_{4} \stackrel{100 \%}{\succ} W_{1} .
$$

That is, the risk level of the enterprise $E_{2}$ takes precedence over $E_{3}$ in a degree of $56.11 \%$, the risk level of the enterprise $E_{3}$ takes precedence over $E_{4}$ in a degree of $98.23 \%$, and the risk level of the enterprise $E_{4}$ takes precedence over $E_{1}$ totally. It means that the enterprise $E_{2}$ has the strongest ability to resist dynamic alliance risk, and the enterprise $E_{1}$ has the weakest ability to resist dynamic alliance risk.

\subsection{Sensitivity Analysis}

Sensitivity analysis is designed to analyze the impact of risk factors for the dynamic alliance on the risk levels of the four enterprises. Four indices, opportunity identification risk $\left(s_{11}\right)$, technology maturity risk $\left(s_{22}\right)$, different location $\left(s_{32}\right)$ and natural environment $\left(s_{52}\right)$ have been selected as they have greater weights.

The sensitivity analysis about opportunity identification risk $\left(s_{11}\right)$ weight as the independent variable has been performed, as shown in Figure 5. Every interval value weight is split into two parts: lower bound weight and upper bound weight. We change these two bound weights from the basic values by rate of $20 \%$, and the corresponding risk level of the four enterprises can be achieved. The risk level curves of the four enterprises are varying with the opportunity identification risk weight. Therefore, the opportunity identification risk is a sensitive factor. 


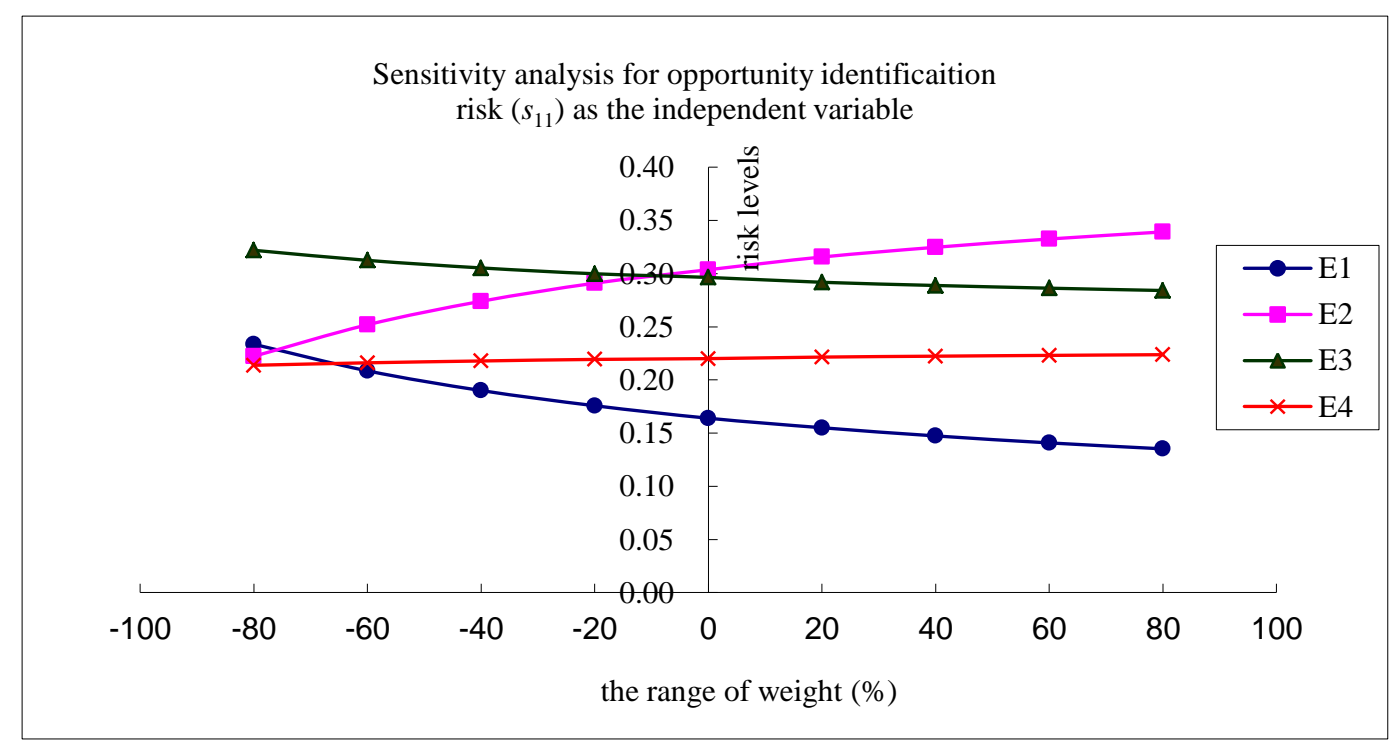

Figure 5. Sensitivity Analysis for Opportunity Identification Risk (s11) Weight

With the increase of opportunity identification risk weight in decision-making factors, the risk level curves of enterprise $E_{1}$ and $E_{3}$ show a downward trend, indicating the risk resistance capabilities of factor $s_{11}$ for both $E_{1}$ and $E_{3}$ are weak. That is, if the dynamic alliance encounters opportunity identification risk, enterprise $E_{1}$ and $E_{3}$ will be more susceptible to its negative effects. The risk level curve of enterprise $E_{4}$ is nearly a horizontal line, which shows $E_{4}$ take well precautions against $s_{11}$. According to the risk level curve of enterprise $E_{2}$, a rising line indicates that $E_{2}$ has a better risk prevention effect for $s_{11}$.

Similarly, the sensitivity analyses for the indices of technology maturity risk $\left(s_{22}\right)$, different location $\left(s_{32}\right)$ and natural environment $\left(s_{52}\right)$ are executed, as shown in Figures 6 , 7 and 8 . Through sensitivity analysis of dynamic alliance, we know that same risk factors have different impacts on enterprises. Consequently, alliance enterprises should pay more attention to the prevention and control of sensitive factors in the risk management. For example, dynamic alliance needs to focus on the risk management for whose risk level decrease when the weights of sensitive factor increase.

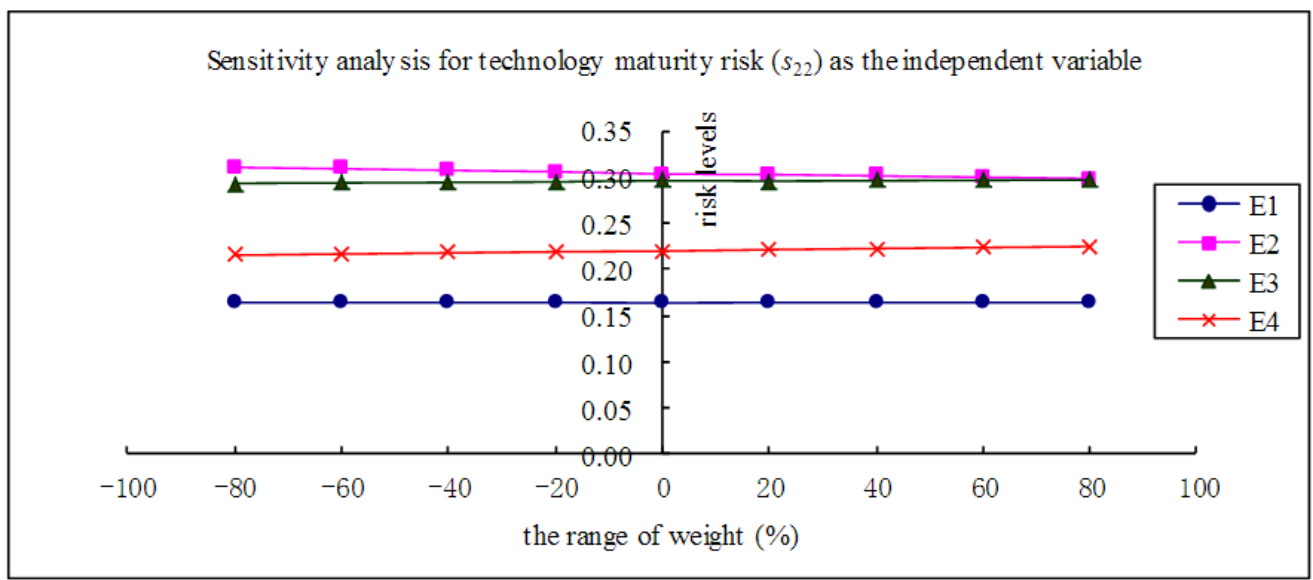

Figure 6. Sensitivity Analysis for Technology Maturity Risk $\left(s_{22}\right)$ Weight 


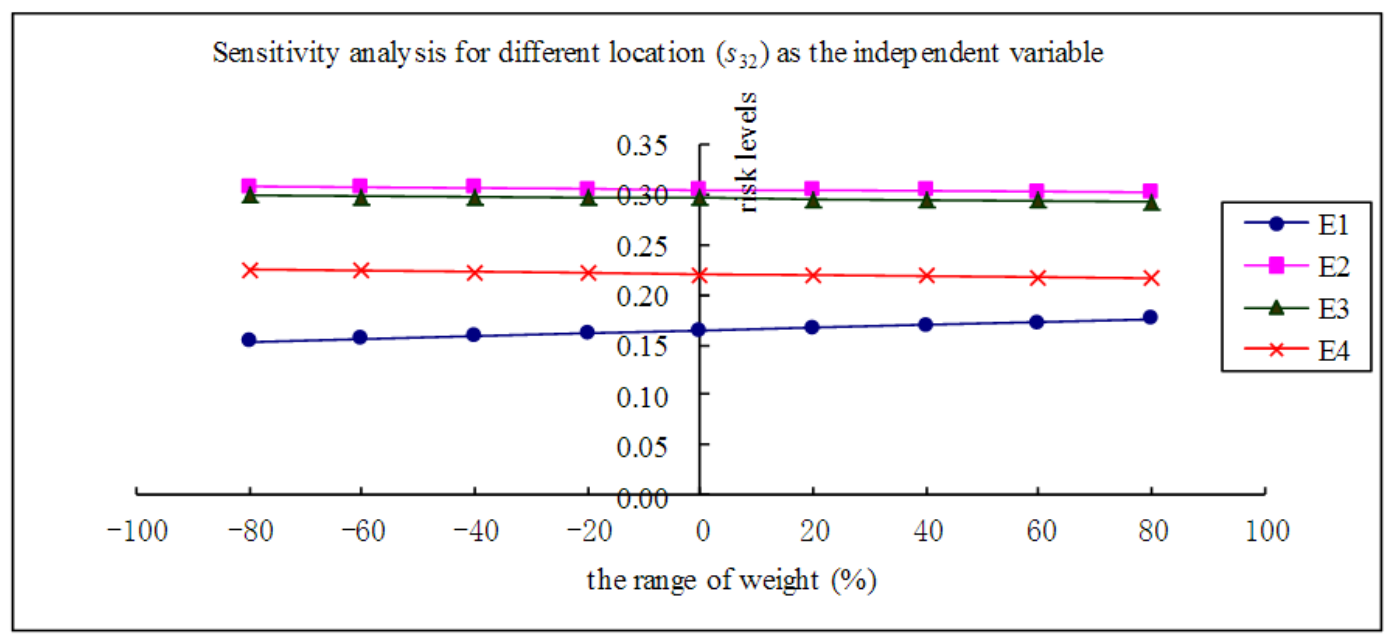

Figure 7. Sensitivity Analysis for Different Location $\left(s_{32}\right)$ Weight

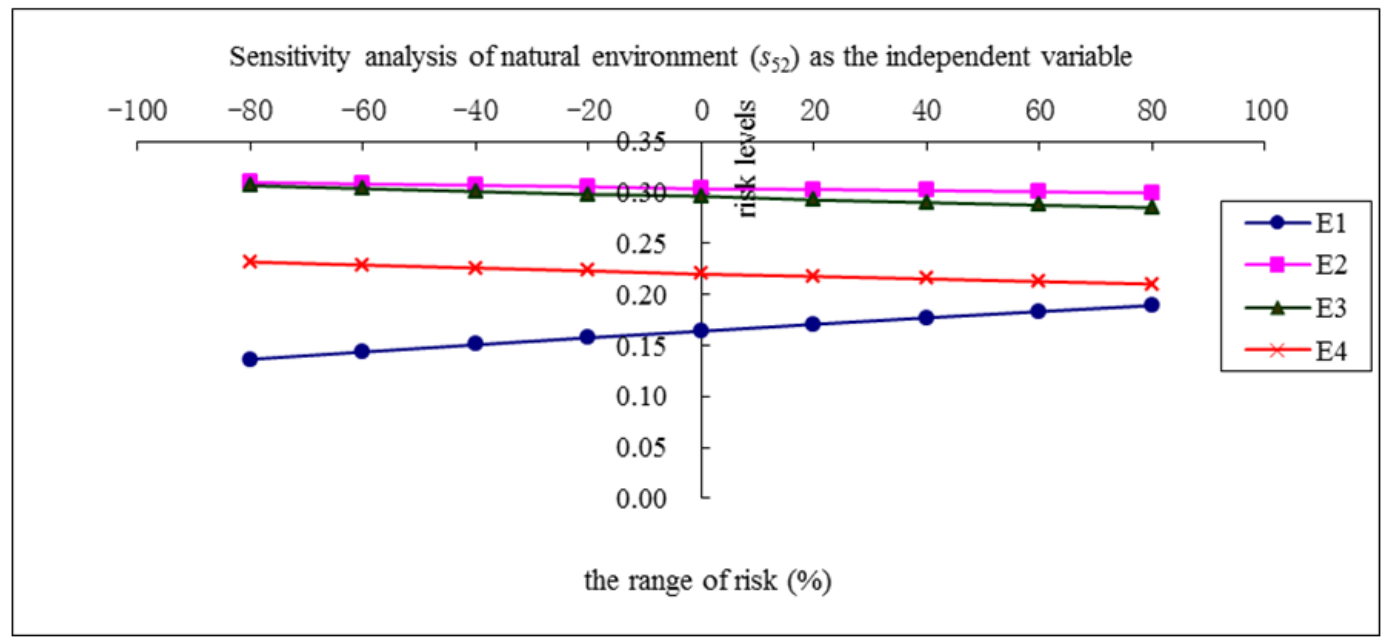

Figure 8. Sensitivity Analysis for Natural Environment $\left(s_{52}\right)$ Weight

\section{Conclusions}

The risk analysis of dynamic alliance will help enterprises to choose a coalition partner and make a reasonable risk management plan, and it is advantageous to keep the stability of the union as well. With the interaction and feedback relationships between criteria and/or indices being considered, an index system for evaluating the risk of dynamic alliance is presented. With the uncertainty and inaccuracy information during the evaluation process being considered, an IV-ANP model is proposed. The local weights of criteria and indices are calculated by goal programming, and the global weights are determined by IV-ANP method. The candidates can be ranked by interval-valued preference order. A risk assessment of dynamic alliance case is given by the proposed method.

An extreme value method for IV-ANP is proposed in this paper. Compared with tradition method, this method achieves interval-valued weights and avoids the loss of information. It should be noted that, the range of weights may be magnified as this approach adopting extreme values. To a certain extent, it may reduce the accuracy. Furthermore, different dynamic alliance has different risk due to different market environment and the characteristics of allied enterprises. 


\section{Acknowledgements}

This work was supported by the National Natural Science Foundation of China (71272161/G021102) and the Fundamental Research Funds for Chinese Central Universities (No. FRF-BR-13-031).

\section{References}

[1] T. P. Munyon, A. A. Perryman, J. Morgante and G. R. Ferris, "Firm relationships: The dynamics of effective organization alliances", Organizational Dynamics, vol. 40, no. 2, (2011), pp. 96-103.

[2] S. R. Holmberg and J. L. Cummings, "Building successful strategic alliances - strategic process and analytical tool for selecting partner industries and firms", Long Range Planning, vol. 42, no. 2, (2009), pp. 164-193.

[3] Y. Li and X. Liao, "Decision support for risk analysis on dynamic alliance", Decision Support Systems, vol. 42, no. 4, (2007), pp. 2043-2059.

[4] R. Venkatesh, V. Mahajan and F. Muller, "Dynamic co-marketing alliances: When and why do they succeed or fail?", International Journal of Research in Marketing, vol. 17, no. 1, (2000), pp. 3-31.

[5] C. Chen, "The effects of environment and partner characteristics on the choice of alliance forms", International Journal of Project Management, vol. 21, no. 2, (2003), pp. 115 - 124.

[6] F. T. Rothaermel and D. L. Deeds, "Alliance type, alliance experience and alliance management capability in high-technology ventures", Journal of Business Venturing, vol. 21, no. 4, (2006), pp. 429460.

[7] H. Delerue and E. Simon, "National cultural values and the perceived relational risks in biotechnology alliance relationships", International Business Review, vol. 18, no. 1, (2009), pp. 14-25.

[8] D. Mukherjee, A. S. Gaur, S. S. Gaur and F. Schmid, „External and internal influences on R\&D alliance formation: Evidence from German SMEs", Journal of Business Research, vol. 66, no. 11, (2013), pp. $2178-2185$.

[9] T. K. Das and B. S. Teng, "A risk perception model of alliance structuring”, Journal of International Management, vol. 7, no. 1, (2001), pp. 1 - 29.

[10] S. Rosenkranz and P. W. Schmitz, „Optimal allocation of ownership rights in dynamic R\&D alliances”, Games and Economic Behavior, vol. 43, no. 1, (2003), pp. 153-173.

[11] W. H. Ip, M. Huang, K. L. Yung and D. Wang, „Genetic algorithm solution for a risk-based partner selection problem in a virtual enterprise", Computers and Operations Research, vol. 30, no. 2, (2003), pp. $213-231$

[12] M. Huang, F. Lu, W. Ching and T. K. Siu, "A distributed decision making model for risk management of virtual enterprise", Expert Systems with Applications, vol. 38, no. 10, (2011), pp. 13208-13215.

[13] P. Lee, H. Chen and Y. Shyr, "Driving dynamic knowledge articulation and dynamic capabilities development of service alliance firms", The Service Industries Journal, vol. 31, no. 13, (2011), pp. 22232242.

[14] M. Huang, W. H. Ip, H. Yang, X. Wang and H. C. W. Lau, "A fuzzy synthetic evaluation embedded tabu search for risk programming of virtual enterprises", International Journal of Production Economics, vol. 116, no. 1, (2008), pp. 104-114.

[15] T. L. Saaty, "Decision Making with Dependence and Feedback: The Analytic Network Process", Pittsburgh, PA: RWS Publications, (1996).

[16] P. Aragonés-Beltrán, F. Chaparro-González, J. Pastor-Ferrando and A. Pla-Rubio, "An AHP (Analytic Hierarchy Process)/ANP (Analytic Network Process)-based multi-criteria decision approach for the selection of solar-thermal power plant investment projects", Energy, vol. 66, (2014), pp. 222-238.

[17] S. Vinodh, R. A. Ramiya and S. G. Gautham, "Application of fuzzy analytic network process for supplier selection in a manufacturing organization”, Expert Systems with Applications, vol. 38, no.1, (2011), pp. 272-280.

[18] G. Kumar and J. Maiti, "Modeling risk based maintenance using fuzzy analytic network process", Expert Systems with Applications, vol. 39, no. 11, (2012), pp. 9946-9954.

[19] G. Yucel, S. Cebi, B. Hoege and A. F. Ozok, "A fuzzy risk assessment model for hospital information system implementation", Expert Systems with Applications, vol. 39, no. 1, (2012), pp. 1211-1218.

[20] Y. Y. Ou, H. Shieh and G. Tzeng, "A VIKOR technique based on DEMATEL and ANP for information security risk control assessment", Information Sciences, vol. 232, (2013), pp. 482-500.

[21] D. Ergu, G. Kou, Y. Shi and Y. Shi, "Analytic network process in risk assessment and decision analysis", Computers \& Operations Research, vol. 42, (2014), pp. 58-74.

[22] X. Zhou and M. Lu, "Risk evaluation of dynamic alliance based on fuzzy analytic network process and fuzzy TOPSIS", Journal of Service Science and Management, vol. 5, (2010), pp. 230-240.

[23] V. Belton and T. Gear, "On a short-coming of Saaty's method of analytic hierarchies", Omega, vol. 11, (1983), pp. 228-230.

[24] P. L. Harker and L. G. Vargas, "The theory of ratio scale estimation: Saaty's analytic hierarchy process", Management Science, vol. 33, (1987), pp. 1383-1403. 
[25] T. L. Saaty, "Decision making with dependence and feedback: The ANP, Pittsburgh: RWS Publications", 4922 Ellsworth Avenue, Pittsburgh, PA 15123, USA, (2001).

[26] I. Yüksel and M. Dağdeviren, "Using the analytic network process (ANP) in a SWOT analysis-A case study for a textile firm", Information Sciences, vol. 177, no. 16, (2007), pp. 3364-3382.

[27] L. Mikhailov, "A fuzzy approach to deriving priorities from interval pairwise comparison judgments", European Journal of Operational Research, vol. 159, no. 3, (2004), pp. 687-704.

[28] Y. Wang and K. Chin, "An eigenvector method for generating normalized interval and fuzzy weights", Applied Mathematics and Computation, vol. 181, no. 2, (2006), pp. 1257-1275.

[29] T. Entani and H. Tanaka, "Interval estimations of global weights in AHP by upper approximation", Fuzzy Sets and Systems, vol. 158, no. 17, (2007), pp. 1913-1921.

[30] K. Sugihara, H. Ishii and H. Tanaka, "Interval priorities in AHP by interval regression analysis", European Journal of Operational Research, vol. 158, no. 3, (2004), pp. 745-754.

[31] Y. Wang and T. M. S. Elhag, "A goal programming method for obtaining interval weights from an interval comparison matrix", European Journal of Operational Research, vol. 177, no. 1, (2007), pp. 458-471.

[32] Y. Wang, J. Yang and D. Xu, "Interval weight generation approaches based on consistency test and interval comparison matrices", Applied Mathematics and Computation, vol. 167, no. 1, (2005), pp. 252273.

[33] S. Lipovetsky and A. Tishler, "Interval estimation of priorities in the AHP", European Journal of Operational Research, vol. 114, no. 1, (1999), pp. 153 - 164.

[34] J. Wu, H. Huang and Q. Cao, "Research on AHP with interval-valued intuitionistic fuzzy sets and its application in multi-criteria decision making problems", Applied Mathematical Modelling, vol. 37, no. 24, (2013), pp. 9898-9906.

[35] C. Kahraman, B. Öztayşi, I. Uçal Sarı and E. Turanoğlu, "Fuzzy analytic hierarchy process with interval type-2 fuzzy sets", Knowledge-Based Systems, vol. 59, (2014), pp. 48-57.

[36] B. Vahdani, H. Hadipour and R. Tavakkoli-Moghaddam, "Soft computing based on interval valued fuzzy ANP-A novel methodology", Journal of Intelligent Manufacturing, vol. 23, no. 5, (2012), pp. 1529-1544.

\section{Author}

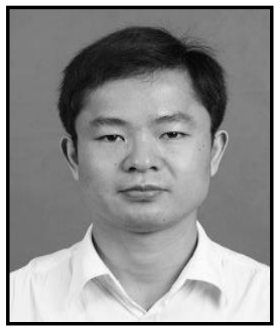

Xiaoguang Zhou, received his $\mathrm{PhD}$ degree in Management Science and Engineering from Beijing Institute of Technology in China. He is currently working at University of Science and Technology Beijing in the Department of Financial Engineering as an Associate Professor. He has published more than 30 papers in journals and conference proceedings. His research interests include operations research, investment decisions and risk analysis, behavioral finance, as well as supply chain management.

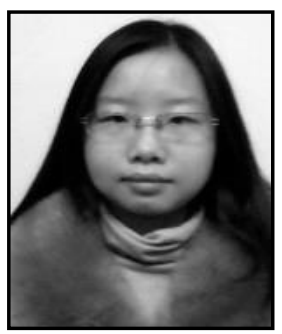

Yanhui Zhou, received her master degree in Management Science and Engineering from University of Science and Technology Beijing in China. Her research interests include decision making, operations research and data mining.

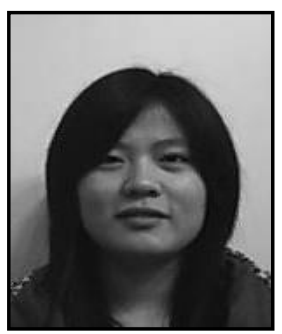

Xiaoxiao Cui received her master degree in Management Science and Engineering from University of Science and Technology Beijing in China. She is currently working at sinosoft Co., Ltd as an engineer. Her research interests include operations research, investment decisions and risk analysis, supply chain management. 